\title{
LGR5 is required for the maintenance of spheroid-derived colon cancer stem cells
}

\author{
XIANG CHEN $^{1^{*}}$, BO WEI $^{1^{*}}$, XIAOYAN HAN ${ }^{2 *}$, ZONGHENG ZHENG $^{1}$, \\ JIANGLONG HUANG $^{1}$, JIANPEI LIU ${ }^{1}$, YONG HUANG ${ }^{1}$ and HONGBO WEI ${ }^{1}$ \\ ${ }^{1}$ Department of Gastrointestinal Surgery, ${ }^{2}$ Central Laboratory, \\ The Third Affiliated Hospital of Sun Yat-sen University, Guangzhou, Guangdong 510630, P.R. China
}

Received January 10, 2014; Accepted April 7, 2014

DOI: $10.3892 / \mathrm{ijmm} .2014 .1752$

\begin{abstract}
Colon cancer stem cells (CCSCs) are involved in colon cancer and promote tumor progression and recurrence. LGR5, a marker for intestinal stem cells (ISCs), is also considered to serve as a marker for CCSCs. However, the precise function of LGR5 in CCSCs is unclear. In this study, we demonstrated that LGR5 was highly expressed in CCSCsenriched HT29 spheroid cells. Downregulation of LGR5 with small interfering RNA (siRNA) decreased the expression of stem the cell markers CD133 and CD44 in HT29 spheroid cells. In addition, silencing of LGR5 inhibited cell proliferation, secondary tumor sphere formation and induced cell apoptosis, and G0/G1 phase arrest in vitro by modulating Bcl-2, Bcl-xL and Bax. Knockdown of LGR5 enhanced chemosensitivity and reduced the invasive ability of HT29 spheroid cells. Moreover, LGR5-siRNA suppressed tumorigenicity of HT29 spheroid cells in vivo. The findings suggested that LGR5 plays a vital role in the maintenance of CCSCs and is a potential therapeutic target for colon cancer.
\end{abstract}

\section{Introduction}

Colon cancer is a common malignancy worldwide with a high incidence of tumor recurrence and metastasis, resulting in cancer-related mortalities (1). Despite advances in therapy, including surgery and chemotherapy, tumor recurrence and metastasis cannot be effectively prevented (2).

Accumulating evidence suggests that a small subpopulation of cancer cells known as cancer stem cells (CSCs), which exist in various types of cancer and are characterized by

Correspondence to: Professor Hongbo Wei, Department of Gastrointestinal Surgery, The Third Affiliated Hospital of Sun Yat-sen University, Tianhe Road 600, Guangzhou, Guangdong 510630, P.R. China

E-mail: drweihb@126.com

*Contributed equally

Key words: LGR5, maintenance, spheroid cells, colon cancer stem cells extensive ability of self-renewal and differentiation, and have a high potential for tumor propagation and therapy-resistance, may contribute to tumor progression, recurrence and metastasis $(3,4)$.

Targeting therapy towards colon CSCs may be a promising approach to eradicating colon cancer more efficiently. Several molecular markers have been previously employed to identify CCSCs, such as CD133, CD44, ALDH and EpCAM (5-8). However, the specificity of these markers are questionable (9). LGR5, a Wnt target gene, was initially identified as a marker of intestinal stem cells (ISCs). LGR5 ${ }^{+}$cells are located at the crypt base and generate various types of differentiated epithelial cells in the intestine to maintain the self-renewal and homeostasis of intestinal mucosa (10-12). CCSCs are considered to originate from normal ISCs (13-15). Conditional deletion of APC exclusively in the LGR5 ${ }^{+}$ISCs of a murine model led to the rapid growth and spread of large adenomas in the small intestine and colon, whereas deletion of Apc in the non-stem cell compartment, resulted in the growth inhibition of adenomas (15). Therefore, LGR5 may also serve as a marker for CCSCs (16). A higher expression of LGR5 has been found in colon cancer and adenomas relative to matched normal mucosa, and is associated with malignant clinicopathological characteristics, suggesting that LGR5 is involved in tumor development and progression $(17,18)$.

Although LGR5 is regarded as a potential marker for CCSCs, little is known concerning its function. We previously reported that colon cancer spheroid cells derived from serumfree culture possessed stem-like properties, including higher proliferative, migratory, invasive, and metastatic ability, and were thus considered CCSCs-enriched models (19). In this study, we detected the expression of LGR5 in these spheroid cells, and assessed its role in CCSCs.

\section{Materials and methods}

Cell culture. The HT29 human colon cancer cell line (ATCC, HTB-38) was maintained in DMEM/F12 with $10 \%$ fetal bovine serum (FBS). For the sphere culture, HT29 cells were grown at a density of $2 \times 10^{6}$ cells $/ \mathrm{ml}$ in $100 \mathrm{~mm}$ ultra-low attachment dishes (Corning Life Sciences, Oneonta, NY, USA) in serum-free DMEM/F12 medium (SFM) containing 2\% B27 (Invitrogen, Carlsbad, CA, USA), $20 \mathrm{ng} / \mathrm{ml}$ epidermal growth factor 
(EGF), $10 \mathrm{ng} / \mathrm{ml}$ basic fibroblast growth factor (bFGF) (both from Peprotech Inc., Rocky Hill, NJ, USA), $5 \mu \mathrm{g} / \mathrm{ml}$ routine insulin (Invitrogen). The cells were incubated in a humidified atmosphere at $37^{\circ} \mathrm{C}$ with $5 \% \mathrm{CO}_{2}$. To induced differentiation in vitro, spheroid cells were cultured in DMEM/F12 supplemented with $10 \%$ FBS for $48 \mathrm{~h}$ and then harvested for assays.

Immunofluorescent staining. Spheroid cells were cytospun onto glass slides, fixed with $4 \%$ paraformaldehyde for $10 \mathrm{~min}$, and permeablized with $0.1 \%$ Triton X-100 for $15 \mathrm{~min}$. Adherent and differentiated cells were cultured on sterile cover slips in 6-well plates for $48 \mathrm{~h}$ and fixed as described above. The cells were incubated with the primary anti-LGR5 Ab antibody (Abcam, Cambridge, UK) at $4^{\circ} \mathrm{C}$ overnight, followed by incubation with secondary DyLight-conjugated anti-rabbit $\mathrm{Ab}$ antibody (Abcam) for $1 \mathrm{~h}$ at room temperature. DAPI (Invitrogen) was used to counterstain the nuclei. Fluorescent images were captured using a Zeiss confocal microscope (LSM-710; Zeiss, Jena, Germany).

Small interfering RNA (siRNA) transfection. siRNA were obtained from GenePharma Co., Ltd. (Suzhou, China). Primer sequences of LGR5-siRNA were: forward, 5'-GCUCCA GCAUCACUUAUGATT-3'; and reverse, 5'-UCAUAAGUG AUGCUGGAGCTT-3'. Dissociated HT29 spheroid cells $\left(5 \times 10^{5}\right)$ were seeded in 6-well plates in SFM. Twenty-four hours later, siRNA were transfected into spheroid cells at a final concentration of $100 \mathrm{nM}$ using Lipofectamine RNAiMAX reagent (Invitrogen) according to the manufacturer's instructions. Spheroid cells were either transfected or not transfected with scrambled siRNA and used as the blank or negative control (NC). The cells were collected for a series of experiments at $48 \mathrm{~h}$ after transfection.

Quantitative RT-PCR ( $q R T-P C R)$. Total RNA was extracted using RNAiso Plus (Takara Bio, Inc., Shiga, Japan) according to the manufacturer's instructions. cDNAs were synthesized using PrimeScript RT reagent kit (Takara) with $1 \mu \mathrm{g}$ total RNA for each sample. The RT-PCR reaction was performed using ABI 7500 Fast (Applied Biosystems, Foster City, CA, USA) with SYBR-Green I reagents (Takara). The cycle condition were: denaturation at $95^{\circ} \mathrm{C}$ for $30 \mathrm{sec}, 40$ amplification cycles at $95^{\circ} \mathrm{C}$ for $3 \mathrm{sec}$ and $60^{\circ} \mathrm{C}$ for $30 \mathrm{sec}$. $\beta$-actin was used as the control. Primer sequences used were: LGR5 forward, 5'-GAGGATCTGGTGAGCCTGAGAA-3'; and reverse, 5'-CATAAGTGATGCTGGAGCTGGTAA-3'; $\beta$-actin forward, 5'-CAACTGGGACGACATGGAGAAA-3'; and reverse, 5'-GATAGCAACGTACATGGCTGGG-3'. The results were analyzed by the $2^{-\Delta \Delta c t}$ method.

Western blotting. Cells were lysed in RIPA buffer with $10 \%$ phenylmethylsulfonyl fluoride. The cell extracts were loaded on 10\% SDS-polyacrylamide gels and transferred onto polyvinylidene fluoride membranes. The membranes were blocked for $1 \mathrm{~h}$ at room temperature with $5 \%$ non-fat milk in TBST, and then incubated with anti-LGR5 (diluted at 1:100; Abgent, San Diego, CA, USA), anti-Bcl-2 (diluted at 1:1,000), anti-Bcl-xL (diluted at 1:1,000), anti-Bax (diluted at 1:1,000) (all from Cell Signaling Technology, Inc., Danvers, MA, USA) at $4^{\circ} \mathrm{C}$ overnight. Following incubation with
HRP-conjugated secondary antibody (diluted at 1:1,000; Santa Cruz Biotechnology, Inc., Santa Cruz, CA, USA ), immuno-complexes were visualized by an enhanced chemiluminescence detection system (Millipore Corp., Billerica, MA, USA). Endogenous GADPH was used for normalization.

Detection of surface markers LGR5, CD133 and CD44 by flow cytometry. Cells were dissociated and washed twice in PBS. Subsequently, cell suspensions were incubated with 1:50 PE-conjugated mouse anti-LGR5 Ab (OriGene, Rockville, MD, USA), or 1:10 PE-conjugated mouse anti-CD133 (Miltenyi Biotec, Bergisch Gladbach, Germany) and 1:10 FITC-conjugated mouse anti-CD44 (BD Biosciences, Franklin Lakes, NJ, USA) antibodies for $20 \mathrm{~min}$ in the dark. The cells were then washed twice in cold PBS with $1 \%$ BSA and resuspended in $300 \mu \mathrm{l}$ cold PBS with $1 \%$ BSA for flow cytometric analysis within $1 \mathrm{~h}$.

Proliferation and chemo-sensitivity assays. Cell proliferation and chemosensitivity assays were determined using Cell Counting kit-8 (CCK-8; Dojindo Laboratories, Kumamoto, Japan). Spheroid cells were dissociated and seeded in 96-well plates at a density of $2 \times 10^{3}$ cells/well in $100 \mu \mathrm{l}$ SFM overnight, and then transfected with $100 \mathrm{nM}$ of indicated siRNA. At $0,1,2,3$ and 4 days after transfection, $10 \mu \mathrm{l}$ CCK-8 reagent was added to each well and the culture was incubated for a further $4 \mathrm{~h}$. The optical density (OD) value in each well was measured by a microplate reader at a wavelength of $450 \mathrm{~nm}$. For chemosensitivity assay, cells $\left(4 \times 10^{3}\right)$ were seeded in each well and treated with various concentrations of 5-Fu $(0,50,100$ and $200 \mu \mathrm{g} / \mathrm{ml})$ or cisplatin $(0,12,24$ and $48 \mu \mathrm{g} / \mathrm{ml})$ (Sigma, St. Louis, MO, USA) $48 \mathrm{~h}$ post-transfection. After incubation for $48 \mathrm{~h}$, a CCK-8 assay was performed and the survival rate of cells was calculated as: $\mathrm{OD}_{\text {treatment }} / \mathrm{OD}_{\text {control }} \mathrm{x} 100 \%$. Experiments were performed in triplicate.

Sphere formation assay. After $48 \mathrm{~h}$ transfection, spheroid cells were dissociated and seeded in 24-well ultra-low attachment plates (Corning Life Sciences) at a density of $2 \times 10^{3}$ cells/well in $500 \mu \mathrm{l} \mathrm{SFM}$ and then grown for a further 7 days. Spheres $>50 \mu \mathrm{m}$ were counted by microscope.

Apoptosis assay. Apoptotic cell rates were measured by Annexin $\mathrm{V}$ and propidium iodide (PI) double staining using an Annexin V/FITC kit (KeyGen, Nanjing, China). Spheroid cells were collected and dissociated $48 \mathrm{~h}$ after transfection, washed with cold PBS containing 2\% BSA, resuspended in binding buffer, and then incubated with Annexin V-FITC and PI for $30 \mathrm{~min}$ in the dark at room temperature. The staining cells were analyzed using FACSCanto II flow cytometer (BD Biosciences).

Cell cycle assay. Spheroid cells were collected $48 \mathrm{~h}$ after transfection. Cells $\left(1 \times 10^{6}\right)$ were fixed with cold $70 \%$ ethanol at $4^{\circ} \mathrm{C}$ overnight, washed with cold PBS, and stained with PI $(50 \mu \mathrm{g} / \mathrm{ml})$ in PBS containing RNase $(50 \mu \mathrm{g} / \mathrm{ml})$ (both from Sigma) in the dark for $30 \mathrm{~min}$. Analyses were performed by FACSCanto II flow cytometer (BD Biosciences).

Invasion assay. Invasion assay was performed using 24-well plate Transwell chambers with $8 \mu \mathrm{m}$-pore polycarbonate filter 


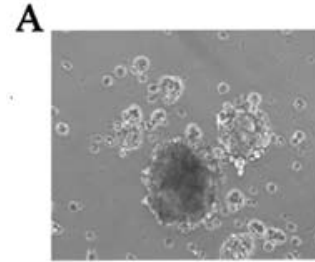

Spheroid

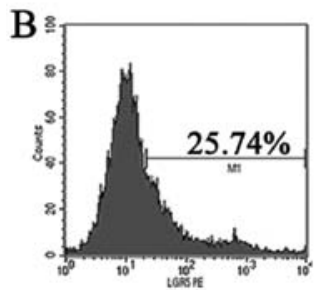

Spheroid

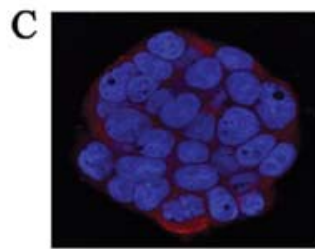

Spheroid

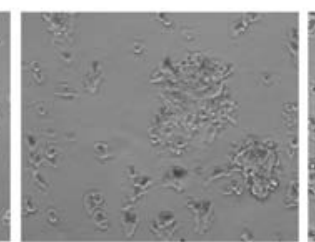

Differentiated

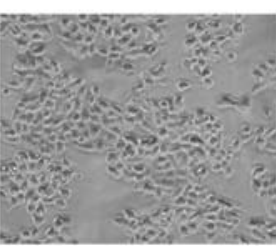

Adherent

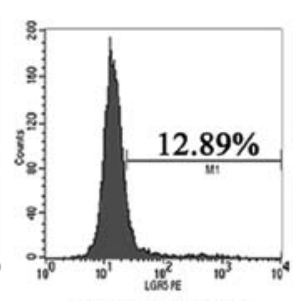

Differentiated

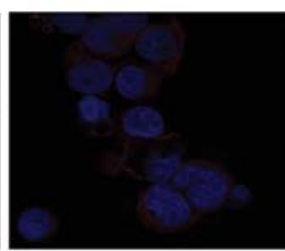

Differentiated

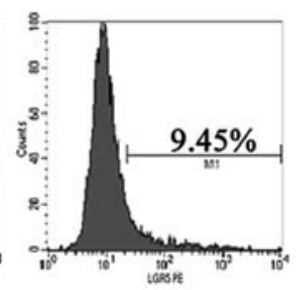

Adherent

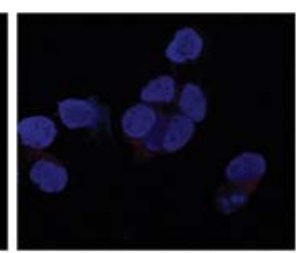

Adherent

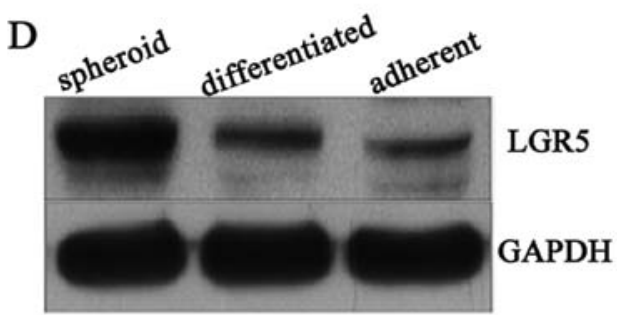

Figure 1. LGR5 expression in spheroid, differentiated and adherent HT29 cells. (A) Morphology of spheroid, differentiated and adherent HT29 cells. When the adherent HT29 cells grew in serum-free DMEM/F12 medium (SFM), they became suspended and turned into large spheres, but after being reseeded in medium containing FBS, the cells were differentiated into adherent cells again (x100). (B) Flow cytometric analysis of LGR5 ${ }^{+}$cell subpopulation in HT29 spheroid, differentiated and adherent cells. (C) Immunofluorescent staining of LGR5 in HT29 spheroid, differentiated and adherent cells. LGR5 staining is red and nuclei are stained in blue. (D) LGR5 protein level in HT29 spheroid, differentiated and adherent cells detected by western blotting.

inserts (Corning Life Sciences). A total of $48 \mathrm{~h}$ post-transfection, spheroid cells were detached and $1 \times 10^{5}$ cells in $100 \mu 1$ DMEM/F12 without FBS were seeded onto the upper chamber coated with Matrigel (BD Biosciences). Then, $600 \mu \mathrm{l}$ DMEM/F12 medium containing 10\% FBS was added into the lower chamber. After incubation for $48 \mathrm{~h}$, the cells on the upper side of the membrane were removed and the cells that migrated to the underside were fixed in $4 \%$ paraformaldehyde, stained with $0.1 \%$ crystal violet and counted in five random fields under microscopy.

In vivo tumorigenesis. Spheroid cells were detached and resuspended in PBS. Cells $\left(2 \times 10^{6}\right)$ in $100 \mu$ l PBS were injected subcutaneously into the flanks of 5-week-old male BALB/C-nu mice (Beijing HFK Bioscience, Beijing, China). When the tumor volume reached $80-100 \mathrm{~mm}^{3}$, the mice were randomly divided into the NC and LGR5-siRNA groups ( $\mathrm{n}=4$ mice per group). The NC and LGR5-siRNA groups were administered intratumoral injection of $50 \mu \mathrm{g}$ scrambled siRNA and LGR5-siRNA, respectively, each week. The diameters of subcutaneous tumors were measured with a caliper every 4 days, and the tumor volume was calculated using the formula: volume $=$ width $^{2} \mathrm{x}$ length $\mathrm{x} 0.5$. Four weeks later, the mice were sacrificed and tumors were extracted and weighed. The experiments were performed in accordance with the Guide for the Care and Use of Laboratory Animals published by the National Institutes of Health.

Statistical analysis. Results were presented as means \pm SD. Statistical analysis was performed using one-way ANOVA or the Student's t-test. The LSD method was used for multiple comparisons. $\mathrm{P}<0.05$ was considered to be statistically significant.

\section{Results}

LGR5 is overexpressed in HT29 spheroid cells. LGR5, also known as GPR49, is a member of the G protein-coupled receptor (GPCR) family of proteins, and enhances Wnt signaling by binding with $\mathrm{R}$-spondin $(10,20)$. Therefore, to obtain comprehensive expression profile in CCSCs, we detected the extra- and intracellular expression of LGR5 in HT29 spheroid 

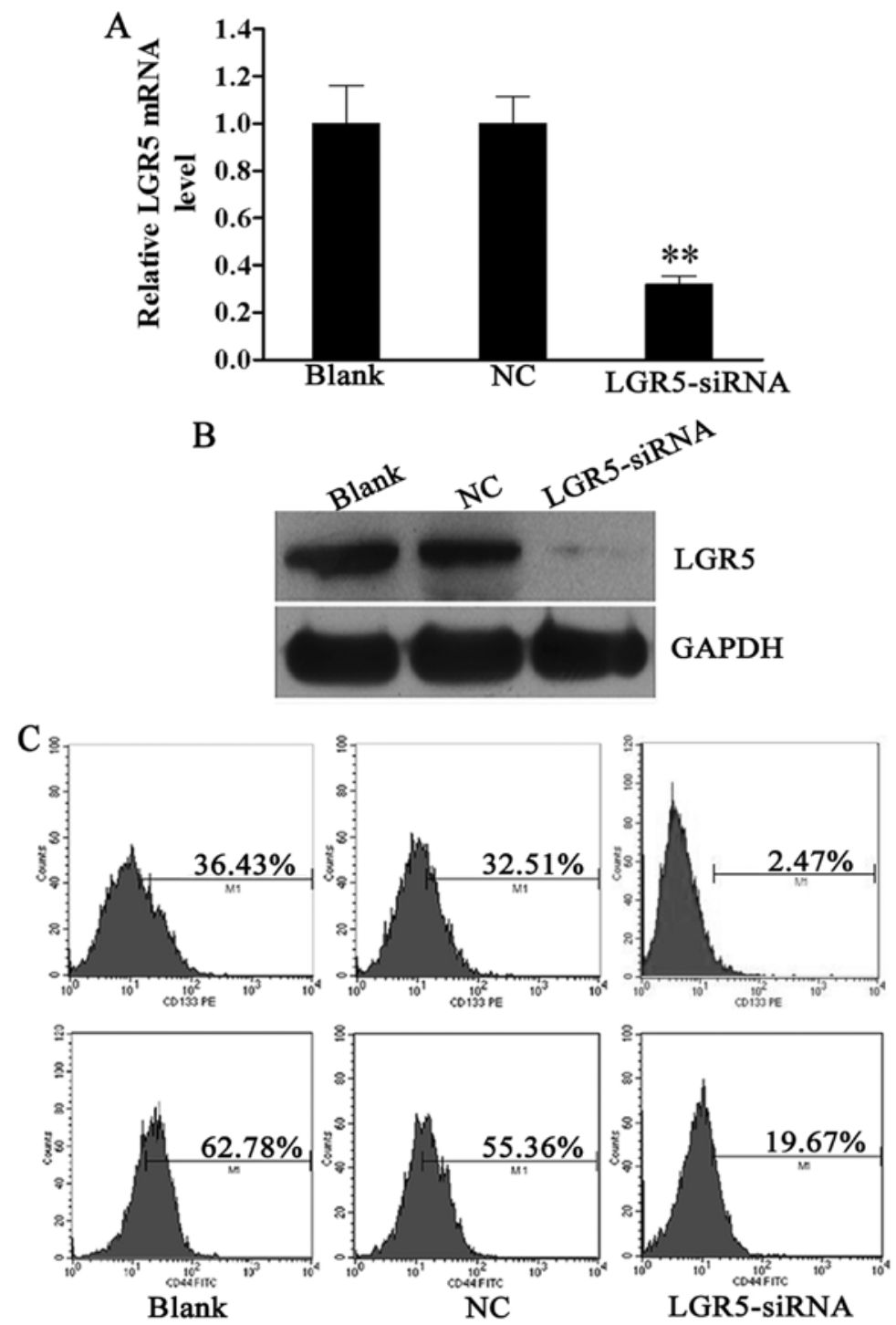

Figure 2. Expression of stem cell markers CD133 and CD44 is downregulated after LGR5 knockdown in HT29 spheroid cells. (A) Relative LGR5 mRNA level of HT29 spheroid cells at $48 \mathrm{~h}$ after small interfering RNA (siRNA) transfection was determined by quantitative RT-PCR (qRT-PCR). (B) Forty-eight hours post-transfection, LGR5 protein expression in HT29 spheroid cells was assessed by western blotting. (C) Expression of CD133 and CD44 was detected by flow cytometry. One of three similar experiments is shown. ${ }^{* *} \mathrm{P}<0.01$ vs. negative control (NC) or blank group.

cells and their counterparts, which were differentiated in vitro and cultured in monolayer (Fig. 1A). Flow cytometric analysis revealed that HT29 spheroid cells contained a high proportion of LGR5 ${ }^{+}$cells, while differentiated and adherent cells had a smaller LGR5 ${ }^{+}$fraction (Fig. 1B). Similarly, compared with adherent counterparts, stronger cytoplastic staining and a higher LGR5 protein level was confirmed by immunofluorescent staining and western blotting in HT29 spheroid cells, respectively, which were significantly attenuated after inducing differentiation in vitro (Fig. 1C and D). These results suggest that LGR5 is associated with dedifferentiation of CCSCs.

Expression of stem cell markers CD133 and CD44 is decreased following siRNA-mediated LGR5 knockdown in HT29 spheroid cells. Since a high expression of LGR5 was detected in HT29 spheroid cells, we investigated the biological function of LGR5 in these cells. siRNA was used to knock down the expression of LGR5 in spheroid cells. LGR5 mRNA was downregulated by $68.2 \%$ in HT29 spheroid cells at
$48 \mathrm{~h}$ after LGR5-siRNA transfection compared to the blank control (Fig. 2A). Moreover, western blotting confirmed that the LGR5 protein expression was also markedly reduced in HT29 spheroid cells transfected with LGR5-siRNA compared with the NC and blank controls (Fig. 2B).

In a previous study, we confirmed that HT29 spheroid cells were rich in $\mathrm{CD} 133^{+}$and $\mathrm{CD} 44^{+}$cells, which represented the subpopulation with stem-like properties (19). The effect of LGR5 on these cell populations was then examined. Forty-eight hours post-transfection, flow cytometric analysis revealed that the percentages of $\mathrm{CD} 133^{+}$and $\mathrm{CD} 44^{+}$cells were decreased in the LGR5-siRNA group (2.47 and 19.67\%), as compared to the NC group (32.51 and 55.36\%) and blank group (36.43 and 62.78\%) (all $\mathrm{P}<0.01$, Fig. 2C). These data reveal that LGR5 plays a key role in sustaining the stemness property of CCSCs.

Downregulation of LGR5 expression impairs survival of HT29 spheroid cells in vitro. In order to investigate the effect 
A

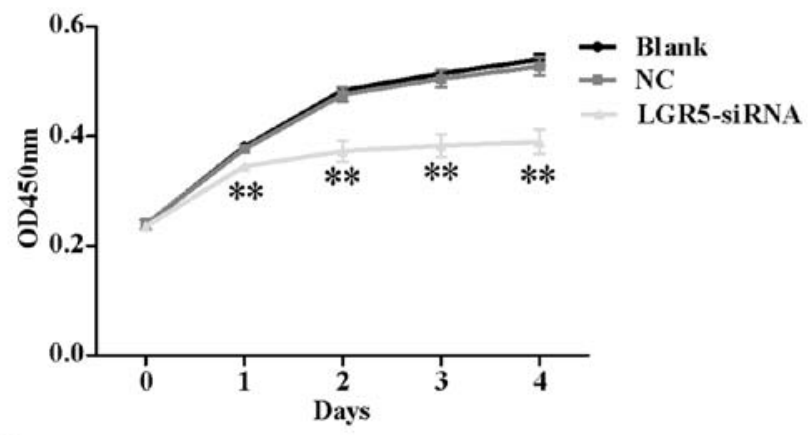

B

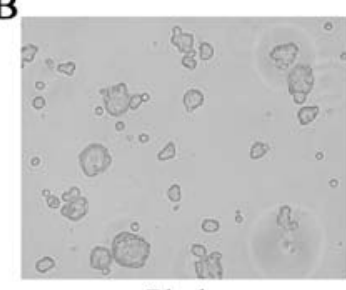

Blank

C

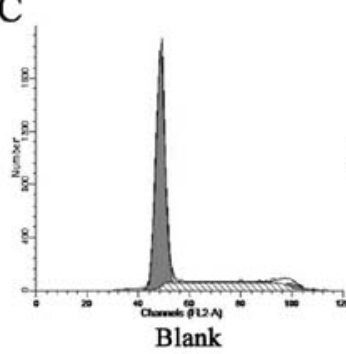

D

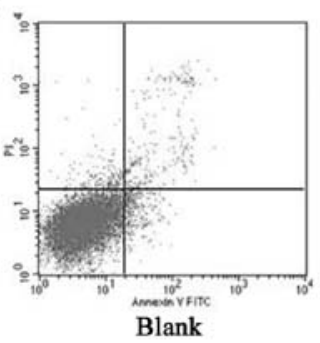

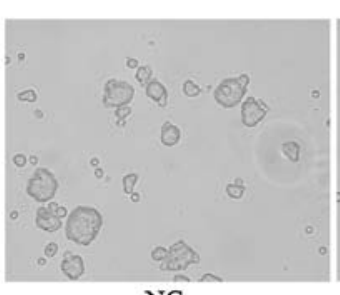

$\mathrm{NC}$
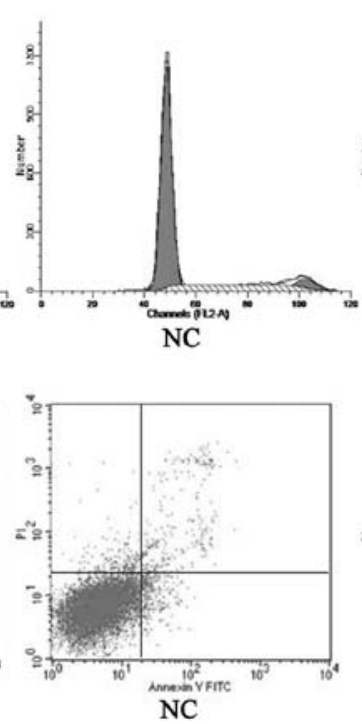

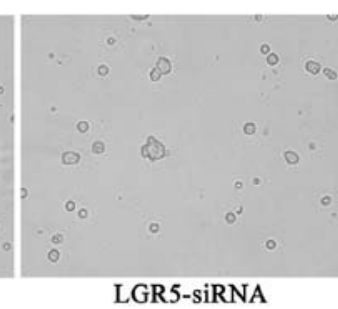

LGR5-siRNA
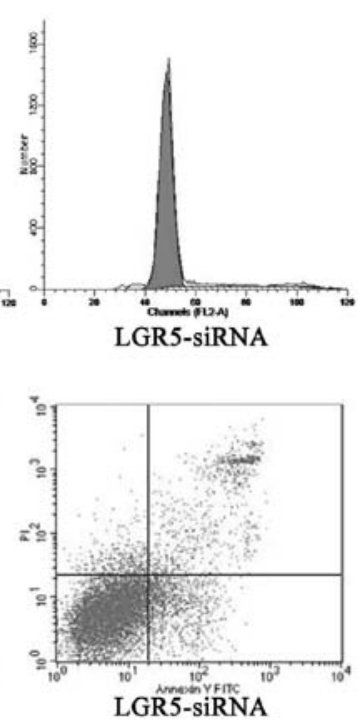

E

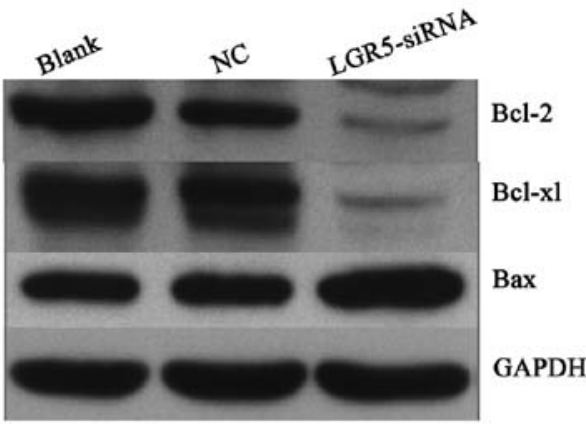

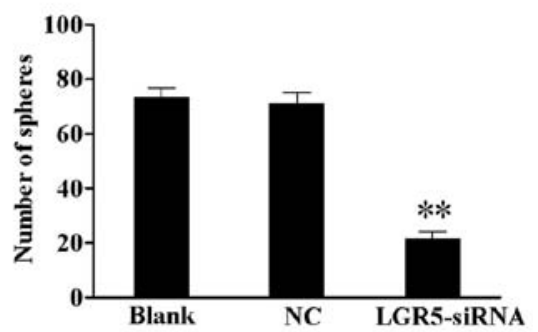
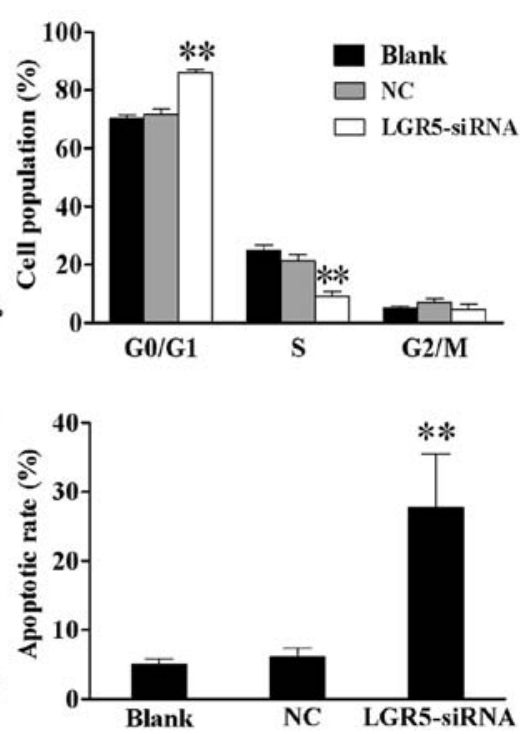

Figure 3. Effect of LGR5 knockdown on survival of HT29 spheroid cells. (A) Growth curves of the blank control, negative control (NC), LGR5-small interfering RNA (siRNA) transfected cells were determined by Cell Counting kit-8 (CCK-8) assay. (B) Tumor sphere formation assay after LGR5 silencing in HT29 spheroid cells (x100). (C) Cell cycle assay assessed by flow cytometry. (D) Cell apoptosis assay determined by flow cytometry of Annexin V/PI staining. (E) Expression of Bcl-2, Bcl-xL and Bax was evaluated by western blotting. Data are presented as means \pm SD from three experiments. ${ }^{* *} \mathrm{P}<0.01$ vs. the NC or blank group.

of LGR5 on survival of CCSCs, proliferation, tumor sphere formation, cell cycle and apoptosis assays were performed in LGR5-siRNA transfected HT29 spheroid cells. CCK-8 assay showed that LGR5-siRNA cell growth was slower than the NC and blank control cells (Fig. 3A). In addition, LGR5 silencing suppressed the self-renewal of HT29 spheroid cells. LGR5-siRNA cells formed smaller and fewer secondary tumor spheres than the NC and blank control cells (Fig. 3B). The cell cycle assay revealed that the percentage of cells at the G0/G1 phase were significantly increased in the LGR5-siRNA group $(86.23 \pm 0.85 \%)$ compared to the NC group $(70.19 \pm 1.35 \%)$ and blank group $(71.84 \pm 1.78 \%)$, while those at $\mathrm{S}$ phase were markedly decreased in LGR5-siRNA group $(9.16 \pm 1.62 \%)$ relative to the NC group $(21.21 \pm 2.13 \%)$ and blank group $(24.77 \pm 2.02 \%)$ $(\mathrm{P}<0.01$, Fig. 3C). On the other hand, the LGR5-siRNA group had a higher apoptotic rate $(27.7 \pm 7.74 \%)$ than the NC group $(6.06 \pm 1.34 \%)$ and blank group $(5.11 \pm 0.77 \%)(\mathrm{P}<0.01$, Fig. 3D). Furthermore, we analyzed the expression of survival-related genes including Bcl-2, Bcl-xL and Bax. Western blotting revealed that the expression of anti-apoptotic Bcl-2 and Bcl-xL genes was downregulated while the expression of the pro-apoptotic Bax gene was upregulated following LGR5 knockdown in HT29 spheroid cells (Fig. 3E). These results show that LGR5 may promote the spheroid cells survival by modulating the intrinsic apoptotic signaling pathway.

Downregulation of LGR5 expression suppresses invasion and increases the chemosensitivity of HT29 spheroid cells. As enhanced invasive ability and chemotherapy resistance are critical features of CSCs, we examined whether LGR5 
A
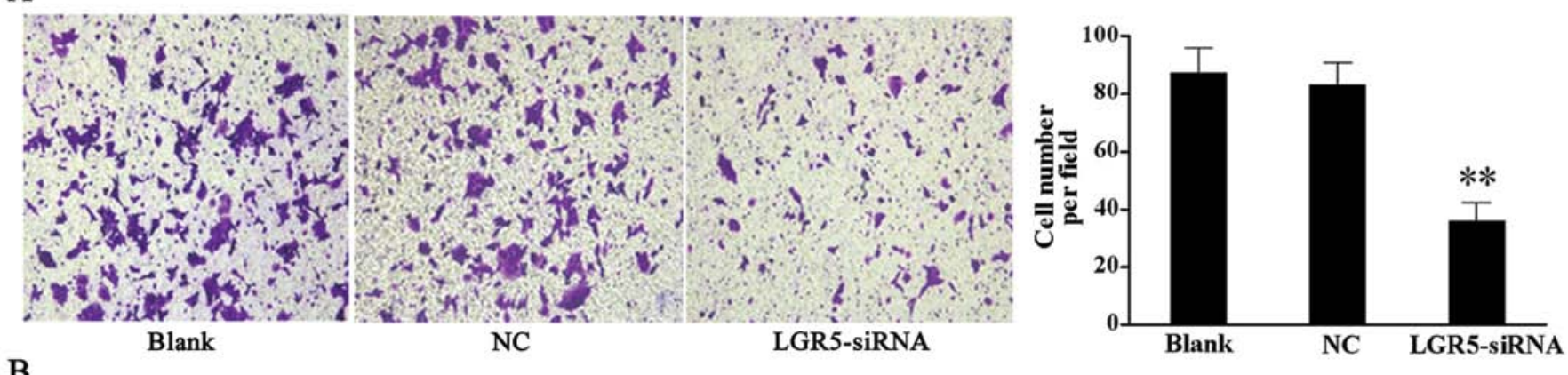

B
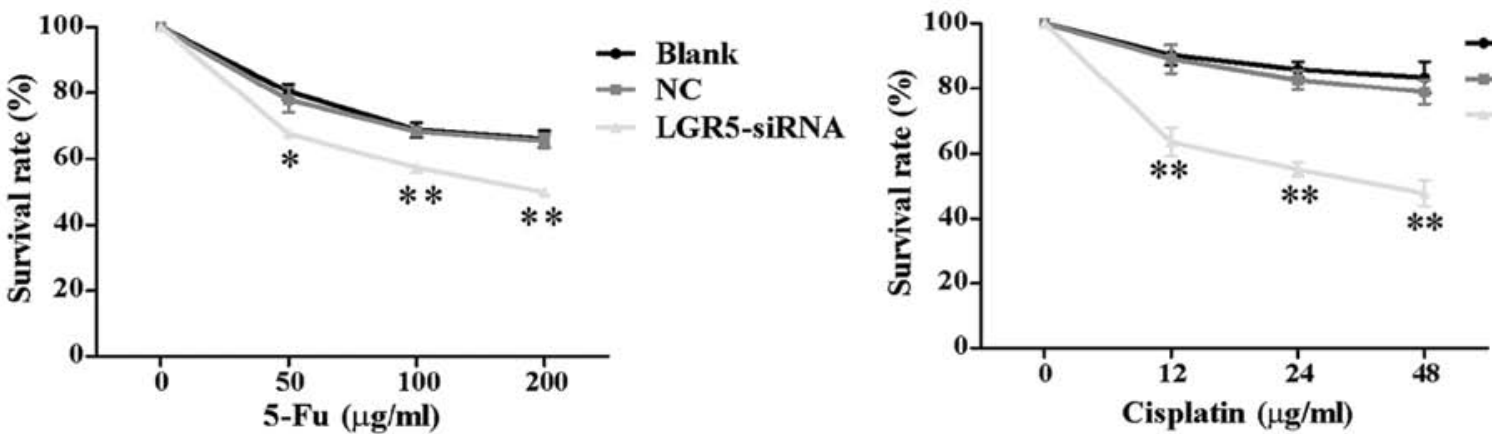

Figure 4. Downregulation of LGR5 suppressed invasive ability and increased chemosensitivity of HT29 spheroid cells. (A) Transwell invasion assay of blank, negative control (NC) and LGR5-small interfering RNA (siRNA) group. Cells that invaded through the Matrigel-coated filter were fixed, stained and counted (x100). (B) After treating with different concentrations of 5-Fu or cisplatin, survival rates of the blank, NC and LGR5-siRNA group were determined by Cell Counting kit- $8(\mathrm{CCK}-8)$ assay. Data are presented as means \pm SD from three experiments. ${ }^{\prime} \mathrm{P}<0.05,{ }^{* *} \mathrm{P}<0.01$ vs. the NC or blank group.

A
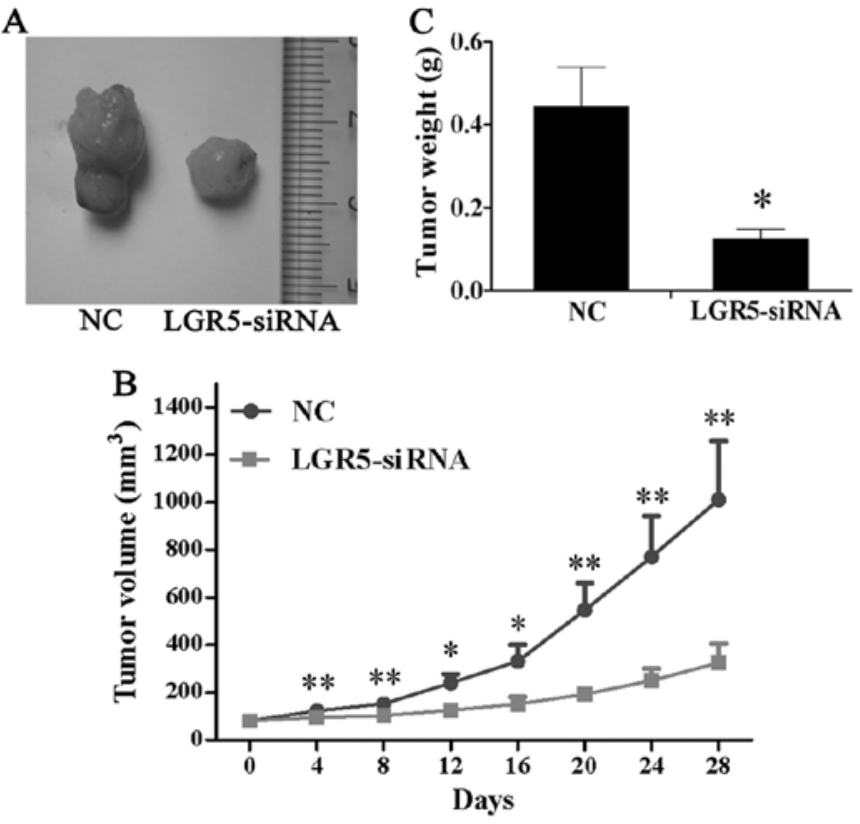

Figure 5. LGR5-small interfering RNA (siRNA) inhabited tumorigenic ability of HT29 spheroid cells in vivo. (A) The image of tumors extracted from mice in the LGR5-siRNA and negative control (NC) groupd. (B) The growth curves showed that LGR5-siRNA resulted in a marked decrease in tumor volume compared with the NC group. (C) Significant reduction in average tumor weight was observed in the LGR-siRNA group, as compared to the NC group. Data are presented as means $\pm \mathrm{SD} .{ }^{*} \mathrm{P}<0.05,{ }^{* *} \mathrm{P}<0.01$ vs. NC group.

affected these features in colon cancer. Results of matrigel invasion assays showed that the number of LGR5-siRNA cells $(35.87 \pm 6.59)$ that invaded the underside of the membrane was significantly less than that of the NC $(83.2 \pm 7.71)$ and blank control cells $(87.27 \pm 8.57)(\mathrm{P}<0.01$, Fig. 4A).

To evaluate the effect of LGR5 on the drug resistance of spheroid cells, we conducted a chemosensitivity assay. Spheroid cells were treated with various concentration of cisplatin and 5 -Fu $48 \mathrm{~h}$ post-transfection. After incubation for further $48 \mathrm{~h}, \mathrm{CCK}-8$ assay demonstrated that the survival rates of LGR5-siRNA transfected cells were significantly reduced compared with the $\mathrm{NC}$ and blank control cells at the respective concentrations (Fig. 4B).

LGR5-siRNA attenuates tumorigenicity of HT29 spheroid cells in vivo. To assess the function of LGR5 for tumorigenicity in vivo, LGR5-siRNA were injected into the subcutaneous tumors in nude mice triggered by HT29 spheroid cells and tumor volume was measured every 4 days. As shown in Fig. 5A and B, mice in the LGR5-siRNA group developed much smaller subcutaneous tumors than those in the NC group. Four weeks after injection, the tumors were excised and weighed. The result showed that the average tumor weight of the LGR5-siRNA group was significantly lower than that of the NC group (Fig. 5C). These findings showed that LGR5-siRNA suppressed tumor growth in vivo in HT29 spheroid cells.

\section{Discussion}

In colon cancer, a small fraction of tumor cells termed CSCs, which possess the ability of multi-differentiation and selfrenewal, are suggested to be responsible for tumor initiation and growth. Therapies targeting CCSCs would likely result in more complete tumor degeneration. Identification 
of CCSCs is therefore a critical step for these targeting therapies. LGR5, known as an intestinal stem cell marker, has been considered a marker for CCSCs $(15,16)$. Previous studies have shown that LGR5 was overexpressed in colon cancer tissue $(17,18)$. However, the expression of LGR5 in CCSCs is unclear. In this study, we identified an increased expression of LGR5 on the surface of spheroid cells by flow cytometry, indicating that these CSCs-enriched colon cancer cells contained a high proportion of $\mathrm{LGR}^{+}$cells. Compared with CD133 expression in spheroid cells we reported previously (19), LGR5 expression may serve as a marker for a smaller heterogeneous population in spheroid cells. More importantly, recent studies have demonstrated that almost all of the $\mathrm{LGR}^{+}$cells isolated from xenografts, generated by the sorted $\mathrm{LGR}^{+}$colon cancer cells, were positive for CD133 and $\mathrm{CD} 166$. In addition, $\mathrm{LGR} 5^{+} / \mathrm{CD} 133^{+}$cells formed more colonies than LGR5 $/$ CD133 ${ }^{+}$cells (21). Findings of recent studies showed that the overlapping expression of putative stem cell markers CD133, CD44 and LGR5 in particular areas of gastric and intestinal mucosa, and concluded that they may be functionally associated $(22,23)$. Our findings that LGR5 knockdown resulted in a decreased expression of CD133 and CD44 in spheroid cells confirm this conclusion, although the underlying molecular mechanism involved remains to be determined. These observations suggest that LGR5 may serve as a robust marker for CCSCs.

LGR5 belongs to the GPCR family and is a target gene of Wnt signaling that regulates tumorigenesis in colon cancer (24). Transformation exclusively occurring in $\mathrm{LGR}^{+}$cells could induce growth of colon adenomas (15). These findings show that LGR5 may be important in CCSCs. This study aimed to assess the function of LGR5 in CCSCs. As such, we examined the intracellular expression of LGR5 in CCSCs-enriched HT29 spheroid cells. The results showed that a high level of LGR5 was detected in the cytoplasm of HT29 spheroid cells, but was markedly decreased when the spheroid cells were induced to differentiate by culturing in FBS-containing medium. These preferential expression patterns suggest that LGR5 was correlated with the maintenance of HT29 spheroid cells. We downregulated LGR5 expression in spheroid cells by siRNA and examined whether it would affect the cells. First, we observed that LGR5 knockdown significantly inhibited the proliferation and secondary tumor sphere formation of HT29 spheroid cells in vitro. In addition, tumor growth in vivo was also suppressed by LGR5 siRNA. In order to clarify the mechanism of these effects, apoptosis and cell cycle assays were performed. The results showed that LGR5 silencing induced apoptosis and G0/ G1 phase arrest in spheroid cells. These data show that LGR5 is essential for survival and the self-renewal of CCSCs, which is consistent with the findings in brain CSCs (25).

High potential for invasion and drug resistance are two important properties of CSCs $(3,26)$. Recent studies have demonstrated that a high LGR5 expression in colon cancer tissues was closely associated with increased lymph node invasion, distant metastasis and poor chemotherapy response $(18,27)$. These findings suggest that LGR5 exerts an effect on the malignant profile of CCSCs. To verify this hypothesis, invasion and chemosensitivity assays were conducted. As expected, LGR5 knockdown significantly reduced invasive ability and increased chemosensitivity in spheroid cells.
The molecular mechanism of LGR5 in modulating CCSCs properties remains to be determined. Results of a recent study showed that Racl-ROS-NF- $\kappa \mathrm{B}$ axis is critical for malignant transformation of $\mathrm{LGR}^{+}$ISC (28). Focal adhesion kinase $(\mathrm{FAK})$, nuclear factor- $\kappa \mathrm{B}(\mathrm{NF}-\kappa \mathrm{B})$, and $\mathrm{c}-$ fos are regulated by LGR5 through the Rho signaling pathway (29). These downstream targets, which are key factors for inflammation and cell adhesion, also play an important role in self-renewal and differentiation and migration of stem cells $(30,31)$. Therefore, the crosstalk between LGR5 and other signaling pathways may contribute to maintaining the function of CCSCs. In this study, we observed that LGR5 silencing altered the expression of Bcl-2, Bcl-xL and Bax in HT29 spheroid cells, suggesting LGR5 is a potential regulator of apoptosis-related genes, although the underlying mechanism involved remains to be determined.

In conclusion, the present study has shown that LGR5 is overexpressed in spheroid-derived CCSCs. LGR5 knockdown suppressed growth, self-renewal, invasion and drug resistance, and induced apoptosis and G0/G1 phase arrest of spheroid cells in vitro, as well as the expression of stem cell markers CD133 and CD44, attenuating their tumorigenicity in vivo. LGR5 is therefore indispensable for the maintenance of CCSCs. Targeting LGR5 may be an effective therapeutic strategy for eliminating colon cancer.

\section{Acknowledgements}

This study was supported by grants from the Project of Science and Technology of Guangzhou City (no. 2011J4100105), the Key Project of Science Foundation of Guangdong Province (no. 10251008901000011), the Project of Science and Technology of Guangdong Province (no. 2009B030801091), the National Natural Science Foundation of China (no. 81000960), and the Fundamental Research Funds for the Central Universities (no. 12ykpy42).

\section{References}

1. Jemal A, Siegel R, Ward E, Murray T, Xu J and Thun MJ: Cancer statistics. CA Cancer J Clin 57: 43-66, 2007.

2. O'Connell JB, Maggard MA and Ko CY: Colon cancer survival rates with the new American Joint Committee on Cancer sixth edition staging. J Natl Cancer Inst 96: 1420-1425, 2004.

3. Dean M, Fojo T and Bates S: Tumour stem cells and drug resistance. Nat Rev Cancer 5: 275-284, 2005.

4. Dalerba P, Cho RW and Clarke MF: Cancer stem cells: models and concepts. Annu Rev Med 58: 267-284, 2007.

5. O'Brien CA, Pollett A, Gallinger S and Dick JE: A human colon cancer cell capable of initiating tumor growth in immunodeficient mice. Nature 445: 106-110, 2007.

6. Du L, Wang H, He L, et al: CD44 is of functional importance for colorectal cancer stem cells. Clin Cancer Res 14: 6751-6760, 2008.

7. Dalerba P, Dylla SJ, Park IK, et al: Phenotypic characterization of human colorectal cancer stem cells. Proc Natl Acad Sci USA 104: 10158-10163, 2007.

8. Huang EH, Hynes MJ, Zhang T, et al: Aldehyde dehydrogenase 1 is a marker for normal and malignant human colonic stem cells (SC) and tracks SC overpopulation during colon tumorigenesis. Cancer Res 69: 3382-3389, 2009.

9. Shmelkov SV, Butler JM, Hooper AT, et al: CD133 expression is not restricted to stem cells, and both CD133+ and CD133- metastatic colon cancer cells initiate tumors. J Clin Invest 118: 2111-2120, 2008.

10. Barker N, van Es JH, Kuipers J, et al: Identification of stem cells in small intestine and colon by marker gene Lgr5. Nature 449: 1003-1007, 2007. 
11. Sato T, Vries RG, Snippert HJ, et al: Single Lgr5 stem cells build crypt-villus structures in vitro without a mesenchymal niche. Nature 459: 262-265, 2009.

12. Snippert HJ, van der Flier LG, Sato T, et al: Intestinal crypt homeostasis results from neutral competition between symmetrically dividing Lgr5 stem cells. Cell 143: 134-144, 2010.

13. Sangiorgi $E$ and Capechi MR: Bmil is expressed in vivo in intestinal stem cells. Nat Genet 40: 915-920, 2008.

14. Zhu L, Gibson P, Currle DS, et al: Prominin 1 marks intestinal stem cells that are susceptible to neoplastic transformation. Nature 457: 603-607, 2009.

15. Barker N, Ridgway RA, van Es JH, et al: Crypt stem cells as the cells-of-origin of intestinal cancer. Nature 457: 608-611, 2009.

16. Merlos-Suárez A, Barriga FM, Jung P, et al: The intestinal stem cell signature identifies colorectal cancer stem cells and predicts disease relapse. Cell Stem Cell 8: 511-524, 2011.

17. Uchida H, Yamazaki K, Fukuma M, et al: Overexpression of leucine-rich repeat-containing $\mathrm{G}$ protein-coupled receptor 5 in colorectal cancer. Cancer Sci 101: 1731-1737, 2010.

18. Takahashi H, Ishii H, Nishida N, et al: Significance of Lgr5(+ve) cancer stem cells in the colon and rectum. Ann Surg Oncol 18: 1166-1174, 2011.

19. Wei B, Han XY, Qi CL, et al: Coaction of spheroid derived stem-like cells and endothelial progenitor cells promotes development of colon cancer. PLoS One 7: e39069, 2012.

20. de Lau W, Barker N, Low TY, et al: Lgr5 homologues associate with Wnt receptors and mediate R-spondin signalling. Nature 476: 293-297, 2011.

21. Kobayashi S, Yamada-Okabe H, Suzuki M, et al: LGR5-positive colon cancer stem cells interconvert with drug-resistant LGR5-negative cells and are capable of tumor reconstitution. Stem Cells 30: 2631-2644, 2012.
22. Wu C, Xie Y, Gao F, et al: Lgr5 expression as stem cell marker in human gastric gland and its relatedness with other putative cancer stem cell markers. Gene 525: 18-25, 2013.

23. Hou NY, Yang K, Chen T, et al: CD133+ CD44+ subgroups may be human small intestinal stem cells. Mol Biol Rep 38: 997-1004, 2011.

24. Vermeulen L, De Sousa E Melo F, van der Heijden M, et al: Wnt activity defines colon cancer stem cells and is regulated by the microenvironment. Nat Cell Biol 12: 468-476, 2010.

25. Nakata S, Campos B, Bageritz J, Bermejo JL, et al: LGR5 is a marker of poor prognosis in glioblastoma and is required for survival of brain cancer stem-like cells. Brain Pathol 23: 60-72, 2013.

26. Brabletz T, Jung A, Spaderna S, Hlubek F and Kirchner T: Opinion: migrating cancer stem cells-an integrated concept of malignant tumour progression. Nat Rev Cancer 5: 744-749, 2005.

27. Hsu HC, Liu YS, Tseng KC, et al: Overexpression of Lgr5 correlates with resistance to 5-FU-based chemotherapy in colorectal cancer. Int J Colorectal Dis 28: 1535-1546, 2013.

28. Myant KB, Cammareri P, McGhee EJ, et al: ROS production and NF- $\kappa \mathrm{B}$ activation triggered by RACl facilitate WNT-driven intestinal stem cell proliferation and colorectal cancer initiation. Cell Stem Cell 12: 761-773, 2013.

29. Kwon MS, Park BO, Kim HM and Kim S: Leucine-rich repeatcontaining G-protein coupled receptor 5/GPR49 activates G12/13-Rho GTPase pathway. Mol Cells 36: 267-272, 2013.

30. Schugar RC, Robbins PD and Deasy BM: Small molecules in stem cell self-renewal and differentiation. Gene Ther 15: 126-135, 2008.

31. Wang SD, Rath P, Lal B, et al: EphB2 receptor controls proliferation/migration dichotomy of glioblastoma by interacting with focal adhesion kinase. Oncogene 31: 5132-5143, 2012. 